\title{
St. Gallen/Vienna 2021: A Brief Summary of the Consensus Discussion on Customizing Therapies for Women with Early Breast Cancer
}

\author{
Christoph Thomssen $^{a} \quad$ Marija Balic $^{b} \quad$ Nadia Harbeck ${ }^{c}$ Michael Gnant ${ }^{d}$ \\ ${ }^{a}$ Department of Gynaecology, Martin-Luther-University, Halle an der Saale, Germany; ${ }^{b}$ Department of \\ Internal Medicine, Division of Oncology, Medical University Graz, Graz, Austria; ${ }^{\circ}$ Breast Center, Department of \\ Obstetrics and Gynecology, University of Munich (LMU), Munich, Germany; ${ }^{\mathrm{d}}$ Comprehensive Cancer Center, \\ Medical University of Vienna, Vienna, Austria
}

\section{Keywords}

Early breast cancer · Neoadjuvant systemic therapy ·

Adjuvant treatment - Endocrine therapy · Chemotherapy ·

Breast surgery · Axillary surgery · Radiotherapy ·

Immunotherapy · Survivorship · Consensus

\begin{abstract}
Because of the COVID-19 pandemic, the 2021 St. Gallen/Vienna Consensus Conference on Early Breast Cancer Treatment Standards had to be held virtually. Despite the challenge of convening global contributors to both the conference itself as well as the important Consensus Panel, the scientific committee and the organizers managed to organize a well-received scientific conference, and also the panel discussion was well received in the worldwide scientific community, as indicated by numerous positive feedbacks already within the first $24 \mathrm{~h}$. The virtual format was unusual, but opened the door for new elements such as Consensus questions proposed from the audience, but also live audience interaction on both days - the Consensus was split into 2 parts in order to accommodate as many time zones globally as possible, leading to almost a doubling of discussion time compared to previous meetings. Also, about 3,400 participants from over 100 countries and all continents came together, including many colleagues who could attend for the first time from world regions with restrictions that so far did not allow the travel to Vienna. Traditionally, the Panel votings and discussions were preceded by 3 days of highlevel live-discussions about the lectures that were available on demand already a week before. Also, all the lectures and
\end{abstract}

live discussions in mini-panels are made available online for at least 6 months (https://www.oncoconferences.ch/events/ bcc-2021/). The traditional panel votings were once more moderated by Eric Winer from Harvard and included interactive elements such as audience votings and audience questions, presented by Michael Gnant. This rapid report by the editors-in-chief of Breast Care summarizes the results of the 2021 international panel votings with respect to locoregional and systemic treatment as a quick news update for our readers and clearly does not intend to replace the official St. Gallen Consensus publication that will follow shortly in Annals of Oncology.

(c) 2021 S. Karger AG, Basel

\section{Introduction}

About 3,400 participants from more than 100 countries and all continents had the opportunity to watch on-demand lectures by some of the most esteemed experts worldwide. Over 3 days, these high-level state-of-the-art and educational lectures addressing local and systemic therapy issues as well as imaging, biomarkers, survivorship, and even COVID-19-related subjects led to great live discussion sessions that gave opportunity for expert discussions of defined topic and - for the first time - for audience questions. A great proportion of sessions were dealing with biology, pathology, and translational research issues, providing not only a great basis for future clinical trials but also defining areas of focused greatest need. Again, a large number of participants from all over the world joined and 
attended the virtual meeting and were actively involved. Once more, the St. Gallen/Vienna meeting remains the most important international breast cancer meeting on European soil from a global perspective.

The Saturday and Sunday consensus panel discussion were once more - virtually - moderated by Eric Winer (USA), who was perfectly supported at Harvard by cochairing Meredith Regan. The other conference co-chairs, Giuseppe Curigliano, Harold Burstein, Michael Gnant, Walter Weber, and Beat Thuerlimann assisted in a variety of ways. Eric Winer mainly moderated the discussion and the voting. More than 160 questions had been developed, prepared, and exchanged by panellists (and the audience who contributed greatly!) upfront in the attempt to reduce the semantic debate and address the most recent controversies in clinically important questions.

It was agreed that clinical trials provide the evidence for general recommendations in clinical decision-making; however, it was also stated, that evidence from randomized clinical trials does not cover all controversies that arise in treating individuals. Thus, expert opinion had to be used when data are lacking. This is the unique feature of the St. Gallen International Consensus.

As an important principle, panellists were asked to consider an "80-percent case" for their decision-making - ignoring individual and or extremely unusual individual patient situations - the message from the Consensus should be generally applicable. Panellists were asked to cast their vote either using three possible answers: yes/no/ abstain or, due to the complexity of some questions, more options were given as possible answers (up to 5). "Abstain" was to be used in case of insufficient data, no personal expertise on the particular issue, or a conflict of interest of a given panellist. After each vote, the answers were summarized in percentages. In this report we summarize the original voting questions and resulting percentages of the St. Gallen/Vienna panel discussion on Saturday March 20 and Sunday March 21, 2021. Abstaining votes and/or very low percentages are not always described in this report.

\section{Imaging}

When patients are planned to receive neoadjuvant systemic therapy, in general magnetic resonance imaging (MRI) should be considered as a standard evaluation. The voting distinguished between patients with HER2-positive and triple-negative breast cancer (TNBC) and ERpositive disease separately. When patients with HER2positive breast cancer or TNBC are planned to receive neoadjuvant therapy, $33 \%$ of the panellists voted that patients should undergo MRI as a standard evaluation, $17 \%$ were against the MRI, and $49 \%$ voted for MRI as a stan- dard evaluation for selected patients who might be candidates for breast conservation. The proportion of panellists voting for MRI changed when ER-positive breast cancers were to undergo neoadjuvant therapy, with $26 \%$ of panellists voting for the MRI as a standard evaluation technique, $23 \%$ against it, and $50 \%$ in favour of MRI for selected patients as candidates for breast conservations.

With respect to the post-excision mammography after breast-conserving surgery, $8 \%$ of the panellists did not find a role for the procedure, $16 \%$ were in favour of postexcision mammography, and $18 \%$ voted in favour where initial microcalcifications were identified by mammography and were treated with breast-conserving surgery. However, in the discussion Peter Dubsky pointed out that this can safely be omitted and patients spared additional examination if the surgical specimen radiographically clearly contains all microcalcifications.

\section{Genetics and Biomarkers}

At the St. Gallen 2021 meeting, biomarkers were discussed in the chapter genetics, pathology, and for indication of adjuvant systemic therapy. Regarding testing of genetic panels for hereditary cancer, $78 \%$ of the panel voted for testing in women if the risk for a hereditary mutation was $>10 \%$ in algorithms based on family history, age at diagnosis, and tumour subtype. Only 10\% recommended testing in all patients, and $12 \%$ suggested testing in all patients $\leq 65$ years. Testing a gene panel including BRCA1 and 2, ATM, BARD1, BRIP1, CDH1, CHEK2, NBN, PALB2, PTEN, STK11, RAD51D, and TP53 was supported by $67 \%$, while $17 \%$ suggested to test BRCA1 and 2 and PALB2. Regarding clinical consequences such as a prophylactic mastectomy in patients with a PALB2 mutation, the panel was undecided with a $50: 50 \%$ vote. Provided that the data from OLYMPIA is going to be clinically compelling, $56 \%$ of the panel would support genetic panel testing in all patients who are potential candidates for adjuvant olaparib.

In a series of questions regarding the importance of prophylactic mastectomy versus routine surveillance according to penetrance of the mutated genes the votes were as follows:

- High penetrance (odds ratio $>3$ ), for example, BRCA1 or 2, PALB2: age 40 years: $85 \%$ support prophylactic mastectomy; age 60 years: $46 \%$ support prophylactic mastectomy and $32 \%$ breast surveillance including MRI.

- Moderate penetrance (OR 2-3), for example, BARD1, CHEK2, CDH1, TP53: age 40 years: 67\% support breast surveillance including MRI and 6\% without MRI; age 60 years: $42 \%$ support breast surveillance including MRI and 35\% without MRI. 
- Low penetrance (OR 1-2), for example, ATM, BRIP1, NF1, RAD51C, RAD51D, FANCC, STK11: age 40 years: $50 \%$ support breast surveillance including MRI and 40\% without MRI; age 60 years: 30\% support breast surveillance including MRI and 64\% without MRI - nobody would recommend prophylactic mastectomy in this situation.

Adding tamoxifen or an aromatase inhibitor (if postmenopausal) to surveillance of patients at increased genetic risk was only supported by a minority of about $5-20 \%$ in the different situations.

\section{Pathology}

The questions for the pathology started with the role of Ki-67. A majority of $62 \%$ agreed with the statement of the International Ki-67 Working Group that in women with ER-positive HER2-negative T1-2 N0-1 breast cancer a low Ki-67 $\leq 5 \%$ would not warrant chemotherapy, whereas a Ki-67 $\geq 30 \%$ would justify chemotherapy. In node-negative ER-positive PR-positive HER2-negative tumours, the majority (42\%) voted for a Ki-67 of at least $30 \%$ for recommending chemotherapy. It should be noted that $36 \%$ of the panel members stated the threshold is not known. In ER-positive HER2-negative breast cancer, Ki-67 should be tested in all cases according to $61 \%$ of the panel, while $30 \%$ would only order $\mathrm{Ki}-$ 67 if chemotherapy is considered and a genomic signature is not available. Sixty-eight percent of the panel supported Ki-67 testing during or after neoadjuvant endocrine therapy (NET) to assess response to NET. Moreover, $70 \%$ of the panel thought that changes in $\mathrm{Ki}-$ 67 after 2 or more weeks of NET can be used to estimate prognosis in women with ER-positive HER2-negative ductal breast cancer.

Regarding the use of multigene signatures to define chemotherapy needs in ER-positive HER2-negative breast cancer with $1-3 \mathrm{~cm}$, the following clinical scenarios were voted on a majority always voting for use in selected patients:

- Male patients: $56 \%$ use in selected patients and 26\% never

- Female patients: $72 \%$ use in selected patients ( $0 \%$ never)

- Premenopausal: $67 \%$ use in selected patients

- Postmenopausal: $64 \%$ use in selected patients

- Node-negative: $64 \%$ use in selected patients

- 1-3 positive nodes: $83 \%$ use in selected patients

- $\quad \geq 4$ positive nodes: $79 \%$ would never order a multigene assay (interestingly, in a spontaneous vote, $38 \%$ of the faculty would order a multigene assay in a 56-year-old woman with a strongly ER- and PR-positive $2.5 \mathrm{~cm}$ tumour with 4,5 , or 6 involved axillary nodes)
- Grade 1: 60\% use in selected patients

- Grade 2: 72\% use in selected patients

- Grade 3: 61\% use in selected patients

- In triple-negative tumours, testing of additional biomarkers was not supported: $93 \%$ disagreed with routine testing of PD-L1 status and $61 \%$ with testing TLIs.

\section{DCIS}

The panel was split on whether radiotherapy can be omitted for DCIS patients older than 70 years ( $58 \%$ yes, $42 \%$ no) or "unifocal no necrosis" ( $47 \%$ yes, $53 \%$ no), but was inclined to allow omission of radiotherapy in situations of low risk (70\%) or G1 DCIS (74\%) or patients over the age of 70 and one of the favourable characteristics $(67 \%)$. The majority of the panel voted for endocrine treatment to prevent recurrence of DCIS (tamoxifen or aromatase inhibitors; 83\%!); most panellists voted to offer standard tamoxifen or aromatase inhibitors (70\%). At least $13 \%$ would explicitly prefer lowdose tamoxifen.

\section{Surgery (Breast)}

The traditional surgical "margin" discussion did not play any role at this year's St. Gallen meeting - the matter appears to be resolved [1]. After "no tumour on ink" was finally firmly established already in 2017 for unifocal residual breast cancers and breast-conserving procedures [2], the 2019 Panel voted with majority that such an approach can also be used for multifocal residual disease [3].

With respect to the timing and sequence of reconstruction and postmastectomy radiotherapy, the panel was completely split about the optimal strategy: delayed reconstruction after radiotherapy $20 \%$, immediate implant in 1 or 2 -stage $23 \%$, immediate autologous reconstruction $25 \%$, delayed immediate (expander) 32\% - with a large number of abstentions, indicating that there is no established standard with respect to this issue. A major change occurred for ipsilateral local recurrence, for which so far mastectomy was considered the standard. In 2021, the majority of the panel endorsed another breast conservation procedure with radiotherapy, if the lead team is more than 5 years (63\%). Factors that would favour a second breast conservation were defined as: low risk (small, luminal A; 81\%); intermediate (5-year) interval since first diagnosis (64\%); the panel was split 50:50 on how the issue should be handled in patients for whom re-irradiation is not an option. 
With regard to surgery after neoadjuvant therapy, the panel clearly declined the idea of omitting surgery in cases of clinical and radiological CR (86\%).

\section{Surgery (Axilla)}

The locoregional standards for the axilla were the "hottest" surgical discussion topics this year - numerous questions regarding this issue were asked to the panel.

After neoadjuvant therapy axillary lymph node dissection (ALND) should be performed when macrometastasis $(73 \%)$ is present; in case of presence of micrometastasis (60\% against) or ITCs ( $89 \%$ against), the majority voted against ALND. These votings demonstrate that the panel is moving towards further de-escalation of axilla surgery after neoadjuvant treatment. However, the "micrometastasis issue" was fiercely discussed, and some prominent surgeons insisted that this would indicate residual disease and therefore ALND should remain mandatory in such cases. In more detail, the panel was asked whether ALND was necessary if 1 out of $3 \mathrm{SN}$ was positive (72\%).

After initial positive lymph node status ( $\mathrm{pN} 1)$ and good clinical response, a biopsied and clipped node would be reliable for avoiding ALND when 1 out of 1 sentinel node is negative (37\%), when 2 out of 2 are negative (7\%), when 3 out of 3 were negative (41\%), or none of the above (14\%), demonstrating that there is no consensus for such situations. For initial pN1 with minor or no response, ALND is necessary according to $82 \%$ of panellists, demonstrating a clear-cut majority. Many experts warned against an - unnecessary? - escalation of radiotherapy when axillary surgery is de-escalated.

The panel was split on targeted axillary dissection in general (60\% no, $40 \%$ yes for "option") and did not endorse targeted axillary dissection for favourable biological subtypes (85\%), but was willing to allow it in $\mathrm{cN} 1$ patients with clipped nodes that had been converted to cN0 (90\%). The panel was split on whether there would be any benefit in removing more than 10 nodes, for example, in cases of heavy nodal involvement ( $>5$ nodes affected; $45 \%$ yes; $55 \%$ no). The panel voted in favour of preservation of intercostobrachial nerves as surgical standard (84\%). The panel could not agree on above which age any axillary surgery can be avoided in case of cN0 $(37 \%>70$ years; $41 \%>80$ years; $5 \%>90$ years; $17 \%$ never).

The panel narrowly endorsed re-sentinel procedures in case of ipsilateral breast recurrence and negative nodes on imaging after previous treatment with breast-conserving surgery and sentinel node mapping (35\% SLNE with frozen section, 33\% SLNE without frozen section, $12 \%$ ALND, 20\% no surgery).

\section{Radiation Therapy}

The 2021 panel was willing to allow hypofractionation in a very progressive way: even for postmastectomy situations, $24 \%$ if regional node irradiation (RNI) is omitted, but $64 \%$ (!) without restriction. For the more general situation of luminal stage 1 or 2 , breast conservation with negative margins, the panel endorsed standard hypofractionation (15-16 fractions) with $72 \%$. Even ultra-short radiotherapy (5 fractions) was considered by $9 \%$, and "any of the options" by $19 \%$. Asked differently, the panel endorsed hypofractionation for postmastectomy radiotherapy (PMRT; 90\%) and RNI (76\%).

In contrast, partial breast irradiation was not endorsed generally ( $0 \%)$, not for lobular ( $80 \%$ no), not for patients with hereditary gene mutations (85\%), not for lymphovascular invasion (87\%), neither for patients under the age of 40 (92\%). RNI was declined for patients with TNBC or HER2-positive breast cancer with pathologically proven complete response (pCR) after neoadjuvant therapy (89\%), but considered mandatory (70\%/65\% always, $26 \% / 30 \%$ in stage 3 ) in stage $2+\mathrm{cN} 1$ TNBC or HER2-positive breast cancer with pCR, respectively. The panel did not think that RNI was necessary with PMRT for TNBC (79\% never) or luminal (95\% never).

Also, the panel was very clear that genomic signatures (Oncotype $\mathrm{DX}^{\circledR}$, MammaPrint ${ }^{\circledR}$, PROSIGNA ${ }^{\circledR}$, etc.) cannot be used to help decide on RNI (92\%) or PMRT (89\%), neither for omission of RT (84\%).

With respect to boost irradiation of the excision site, the panel was generally in favour of it in situations of higher risk (young age, G3, EIC+ TNBC, HER2pos), but some panellists thought boost should be given to every patient after breast conservation (20\%). The panel was, however, clear (89\%) that not all cases of DCIS needed boost, but was split on DCIS and age $>50$ : $45 \%$ yes, $55 \%$ no). However, the panel endorsed boost for DCIS in all high-risk situations (e.g., necrosis, close margins, larger size; $65 \%$ yes).

The panel did not endorse omission of radiotherapy after breast conservation in women older than 70 in general (74\%), node-positive disease (90\%), or tumours $>2.5$ $\mathrm{cm}(80 \%)$, but was willing to omit radiotherapy in patients with tumours $<2.5 \mathrm{~cm}$ and low grade or low genomic score (88\%).

The panel showed uncertainty when asked whether radiotherapy can replace surgery (when 2 of 3 nodes are positive $-38 \%$; 1 out of 3 positive $-52 \%$ ), demonstrating some movement in this area. However, Philip Poortmans warned specifically against "sloppy" radiotherapy extensions ("high tangents") since this would give away modern technological achievements (better tailoring) of contemporary radiotherapy. 
The panel was much more inclined to allow axillary radiotherapy instead of ALND in initially clinically negative axilla in patients without macroscopic nodal involvement (1 of 3 SLN with micrometastasis: $72 \%$; 1 of 3 SLN with ITCs: 88\%).

Also, tumour subsets and available systemic therapies were not endorsed as triggering axillary radiotherapy instead of ALND (HER2 with TDM-1: 68\% no, ER-positive with endocrine therapy: $60 \%$ no, TNBC with capecitabine: $77 \%$ no).

\section{Adjuvant and Neoadjuvant Therapy}

Traditionally, the votes on (neo)adjuvant therapy are the core piece of the St. Gallen Consensus conferences [4]. This year, some questions were discussed several times resulting in sometimes different or even seemingly contradictory votings. We will report on these as it was given without any comment.

\section{Neoadjuvant Therapy}

The block of questions on neoadjuvant treatment began with a discussion of the fact that more than 10 years ago national agencies proposed $\mathrm{pCR}$ as a surrogate endpoint for drug approval in early-stage breast cancer. The panellists were asked whether regimens improving pCR rates should become standard. Only $10 \%$ of the panellists voted in favour of this approach if the regimens achieved a remarkable improvement in pCR rates $(50 \%$ higher than control) and $83 \%$ of panellists voted in favour of neoadjuvant $\mathrm{pCR}$ rate as encouraging; however, only improvement of event-free survival and overall survival endpoints should lead to definition of "standard" regimens. Only $40 \%$ of the panellists agreed with the approach that some national guidelines recommend a decision towards neoadjuvant treatment for all patients based on diagnostic biopsy. Sixty percent of panellists voted against this as being an appropriate approach for all patients.

For postmenopausal women with lower grade and/or low-risk genomic signature cancers, a high majority of panellists with $98 \%$ did not agree that chemotherapy was a treatment of choice for these patients. In addition, 73\% of panellists voted in favour of performing genomic assays on core biopsies to select patients with ER-positive breast cancers for neoadjuvant chemotherapy versus endocrine therapy.

In a case of HER2-positive node-negative breast cancer, $84 \%$ of the panellists do not see a need for anthracyclines in patients receiving taxane-based chemotherapy and anti-HER2 antibodies. However, in the case of node- positive disease $54 \%$ of the panel would consider anthracyclines as needed in the treatment. Furthermore, in clinically positive axillary lymph nodes, according to $62 \%$ of the panel, the treatment should contain anthracyclines, and an additional $23 \%$ would consider platinum- and pertuzumab-containing treatment as preferred. In stage 2 and 3 clinically node-negative HER2-positive disease, patients should receive anthracyclines and pertuzumab in addition to taxane and trastuzumab according to $35 \%$ or pertuzumab and platinum according to another $27 \%$.

When treating triple-negative disease with neoadjuvant chemotherapy, 90\% would not add an immune checkpoint inhibitor to the neoadjuvant treatment and $81 \%$ do not see PD1/PDL1 testing affecting the recommendation for the use of immune checkpoint inhibitors in the neoadjuvant therapy.

\section{Postneoadjuvant Treatment}

In the case of HER2-positive disease with pCR after neoadjuvant therapy, $65 \%$ of the panellists consider the baseline stage and tumour subtype to still influence the prognosis. Therefore, when a pCR is achieved in $\mathrm{cN}+$ HER2-positive breast cancer with pertuzumab/trastuzumab-based treatment, 56\% would consider trastuzumab and pertuzumab as the adjuvant treatment of choice irrespective of baseline stage; however, 22\% would consider pertuzumab and trastuzumab only in case of baseline stage 3 and 22\% would consider trastuzumab only as adjuvant treatment. Following a pCR with trastuzumab/pertuzumab-based therapy in case of cN0 HER2-positive disease, 70\% would consider trastuzumab alone as adjuvant treatment, $12 \%$ would consider pertuzumab and trastuzumab in case of baseline stage 1 or 2 , and $18 \%$ would consider both pertuzumab and trastuzumab with baseline stage 2 only. The majority of the panellists ( $89 \%$ ) would offer trastuzumab-emtansine to all patients with residual disease and $77 \%$ would offer trastuzumab emtansine also to patients with excellent clinical response to anti-HER2 treatment and $<5 \mathrm{~mm}$ residual disease.

If $\mathrm{pCR}$ is achieved in TNBC, $85 \%$ voted against the use of immune checkpoint inhibitors in the adjuvant setting. In contrary, $87 \%$ would offer adjuvant capecitabine to patients with triple-negative patients after neoadjuvant treatment having residual disease.

The only $100 \%$ vote was achieved for patients with ERpositive disease after neoadjuvant endocrine treatment and not achieving $\mathrm{pCR}$ when panellists were asked whether these patients should be offered chemotherapy if they had excellent clinical response and node-negative residual cancer. If there were $\geq 4$ residual lymph nodes $91 \%$ of the panellists would offer adjuvant chemotherapy and if 
there were any residual positive lymph nodes, $52 \%$ would consider offering adjuvant chemotherapy. In case there was $>5 \mathrm{~cm}$ residual tumour in the breast, $77 \%$ would offer adjuvant chemotherapy to patients, and in case of baseline high-grade tumour and/or intermediate-range genomic signature $72 \%$ would offer adjuvant chemotherapy. However, only $22 \%$ agreed that if there was residual disease after NET, no chemotherapy should be considered at all, since the decision was already made.

\section{Adjuvant Endocrine Therapy}

The appropriate threshold ( $\geq 1$ vs. $\geq 10 \%$ ) for recommending adjuvant endocrine therapy in ER-positive breast cancers tested by IHC is a matter of debate; the panel voted 50:50. In a systematic fashion, conditions of tumour biology (molecular type) and tumour size were checked for a threshold being appropriate to offer an adequate adjuvant therapy. For patients with ER-positive HER2-negative breast cancer including luminal A-like and B-like lesions, the majority opted for recommending endocrine adjuvant therapy with any tumour size including microinvasive cancer (59\%) or with at least $1 \mathrm{~mm}$ size (21\%). In ER-positive HER2-positive disease, most panellists would offer anti-HER2 therapy in tumours that scale at least $5 \mathrm{~mm}(51 \%)$ or $6 \mathrm{~mm}(23 \%)$; however, $26 \%$ would offer anti-HER2-therapy also in smaller lesions. For patients with ER-negative HER2-positive breast cancer the recommendation was less clear: many panellists voted for tumour size $5 \mathrm{~mm} \mathrm{(33 \% )}$ or $6 \mathrm{~mm} \mathrm{(19 \% )} \mathrm{as} \mathrm{minimum} \mathrm{to}$ justify an HER 2 therapy; however, in total, $48 \%$ argued to offer therapy also in tumours smaller than $5 \mathrm{~mm}$ or microinvasive only. In TNBC, the majority of the panellists (46\%) considered $5 \mathrm{~mm}$ as an optimal threshold to indicate adjuvant or neoadjuvant chemotherapy, but $35 \%$ of the panellists would offer adjuvant chemotherapy also for patients with tumours smaller than $5 \mathrm{~mm}$ and some (9\%) even in microinvasive disease.

\section{Adjuvant Endocrine Therapy in Premenopausal Patients}

The contribution of chemotherapy-induced ovarian function suppression (OFS) to the total effect of chemotherapy in premenopausal, ER-positive early stage with favourable biological features was estimated by most panellists (42\%) as at least $75 \%$ with a broad range between no contribution and $100 \%$ of the effect. If OFS is used, the panel's vote was ambiguous ( $47 \%$ in favour) considering routine monitoring of estradiol levels as mandatory. There was nearly agreement (94\%) that premenopausal patients with ER-positive HER2-negative breast cancers of sufficient risk to warrant chemotherapy should also receive OFS if they remain premenopausal after chemotherapy treatment [5].

For premenopausal women with node-negative breast cancer and recurrence score 16-25 or other low-range genomic signature, most panellists (53\%) considered OFS with tamoxifen or aromatase inhibitor as adequate therapy, only $25 \%$ voted in favour of chemotherapy plus endocrine therapy. For premenopausal women with 1-3 positive lymph nodes and recurrence score $<25$ or other low-range genomic signature, the voting was inconclusive; $30 \%$ would prefer chemotherapy and oral endocrine therapy, further $26 \%$ favoured chemotherapy, but considered endocrine treatment also as reasonable, the remaining favoured endocrine therapy only.

In general, most panellists supported offering OFS to premenopausal patients with stage 2 disease (71\%), particularly in patients younger than 40 years (94\%). However, independent from a prior adjuvant chemotherapy, the majority (52\%) voted to recommend OFS only if the patient presents with high-risk features (age $<40$ years, node-positive, high Ki-67, luminal B-like, intermediateor higher-risk genomic signatures), although a relevant proportion of the panellists (43\%) would recommend OFS to all patients.

\section{Extension of Endocrine Therapy in Premenopausal Patients}

One section focused on the extension of adjuvant endocrine therapy beyond 5 years. For node-positive ERpositive HER2-negative patients a majority ( $87 \%$ ) considered a prolonged endocrine therapy as indicated, 34\% voted for additional $2-3$ years, $53 \%$ for 10 years. There was a debate on how to treat high-risk premenopausal patients who had finished 5 years of OFS plus tamoxifen with regard to the type endocrine therapy extension: $41 \%$ of the panellists would offer OFS (if still premenopausal) plus aromatase inhibitor and $45 \%$ voted to offer tamoxifen only for 5 years.

\section{Adjuvant Endocrine Therapy in Postmenopausal Patients}

In general, most of the panellists agreed that there were no postmenopausal patients with stage $\leq 2$ ER-positive HER2-negative breast cancer with low-risk signatures (e.g., recurrence scores $\leq 25$ ) who should receive chemotherapy $(79 \%)$. However, in a more detailed discussion, only $51 \%$ would withhold chemotherapy and many would offer chemotherapy for higher anatomical stages like pT3pN1 or $>3$ infiltrated lymph nodes (43\%). The public 
vote, interestingly, was different: only $27 \%$ would withhold chemotherapy and a majority offer chemotherapy for higher stages (61\%).

Discussing whether for patients with high anatomical stage (e.g., stage 3) postmenopausal ER-positive HER2negative breast cancer, the preferred treatment would be combined chemotherapy and endocrine therapy (as opposed to endocrine therapy alone), a majority agreed for patients with very high stages, for example, $\geq 10$ infiltrated lymph nodes (96\%), regardless of biomarkers (68\%), even with recurrence score $<25(58 \%)$. However, for some situations the majority rejected the idea of additional chemotherapy: low grade (G1) or low Ki-67 (63\%), recurrence score $<11(61 \%)$, grade 1 or 2 or lobular breast cancer $(52 \%)$.

\section{Adjuvant Chemotherapy}

Numerous regimens are used in adjuvant therapy. In women with ER-positive breast cancer receiving adjuvant chemotherapy for stage 1 or node-negative breast cancer, the preferred regimen is anthracycline/cyclophosphamide/taxane combination (standard or dose-dense: $40 \%$ ). However, a substantial proportion of the panellists voted for shorter regimens such as ECx4 or DocCx4 (46\%). With higher stage or higher tumour burden almost all panellists (96\%) would recommend standard or dosedense anthracycline/cyclophosphamide/taxane combination (dose dense 39\%).

\section{HER2-Positive Breast Cancer Adjuvant Therapy}

Patients with node-negative, HER2-positive breast cancers should not receive adjuvant pertuzumab in addition to trastuzumab (94\%). There was a majority $(64 \%)$ that recommended offering patients with HER2-positive breast cancers to receive adjuvant neratinib after trastuzumab/pertuzumab and/or trastuzumab emtansinebased as (neo)adjuvant therapy.

Facing the discussion about the role of anthracyclines in severe toxicities such as congestive heart failure, the panellists were still convinced that there might be patients with HER2-positive breast cancer who may receive a sequential anthracycline therapy as part of their chemotherapy regimen [5] even when receiving anti-HER2 treatment in combination with non-anthracycline chemotherapy (76\%).

For women with stage 1 HER2-positive breast cancers, the majority (69\%) did not consider trastuzumab-emtansine to be routinely used instead of paclitaxel/trastuzumab-based therapy; however, $32 \%$ stated that there might be circumstances that justify the use of T-DM1.

\section{New Drugs}

\section{Abemaciclib}

With only recently published data the use of adjuvant abemaciclib was discussed. The result was nearly equivocal: a slight majority of the panellists (54\%) voted in favour of recommending abemaciclib in ER-positive HER2negative patients with more than 3 positive axillary lymph nodes. In addition, other possible situations were discussed, and for patients with 1-3 positive lymph nodes or other factors of an unfavourable prognosis as G3, T3, or high Ki-67 the majority voted against the application of abemaciclib (54\%). Actually, there was also no majority (40\%) that would support Ki-67 for indication of CDK4/6directed therapy.

\section{Immune Checkpoint Inhibitors in TNBC}

There was $90 \%$ agreement of the panellists that patients with stage 2 or 3 TNBC, not treated in the neoadjuvant setting, but receiving adjuvant chemotherapy, should not receive PD1/PDL1-targeted treatment with an immune checkpoint inhibitor.

\section{PARP Inhibitor in TNBC}

In expectation of first data for the adjuvant use of a PARP inhibitor, a relative majority (48\%) considered an iDFS improvement of at least $5 \%$ at 3 years as sufficient to recommend olaparib to patients with BRCA1/2-associated breast cancer. However, by further $25 \%$ an iDFSimprovement of at least $10 \%$ at 3 years was requested.

\section{Survivorship}

This time at the St. Gallen conference special focus was given to survivorship topics. A first important question referred to significant genitourinary or sexual health symptoms during the aromatase inhibitor treatment and when these are not alleviated with the use of lubricants and moisturizers, whether the panellists would recommend the use of intravaginal oestrogens as safe way to relieve symptoms. Fifteen percent answered with yes, the great majority with $72 \%$ were in favour of the use while explaining that there is no reasonable confidence in safety, and $11 \%$ would not recommend intravaginal oestrogens due to safety concerns. In discussion it was pointed out that systemic levels of oestrogens do not get markedly elevated and the treatment is mostly only for a limited time and in patients with lower risk, and after discussing the risks and benefits with patients there is no 
reason for great safety concerns. The use of cold caps may routinely be offered to patients receiving chemotherapy which induces alopecia, and $69 \%$ of panellists were in favour of their use. The use and endorsement of several integrative therapies as a support for management of symptoms and adverse events in breast cancer survivors was discussed, and the panellists were able to only vote in favour of one of the offered approaches; $43 \%$ voted in favour of aerobic exercise, $20 \%$ in favour of acupuncture and weight loss, respectively, $14 \%$ in favour of weight bearing exercises, and $2 \%$ for vitamin supplements. Eighty percent of all panellists were convinced that meditation and mindfulness-based stress reduction should be recommended to breast cancer patients to reduce depressive symptoms. While none of the panellists would suggest breast cancer survivors to completely avoid consumption of alcohol in order to reduce breast cancer risk of recurrence, $57 \%$ would recommend to limit alcohol consumption to $\leq 1$ alcoholic drink per day, $6 \%$ to $\leq 2$ drinks per day, and $14 \%$ were for no restrictions.

\section{Oligometastatic Breast Cancer}

With some controversy, the panel still endorsed for the first time curative intention for oligometastatic breast cancer, for example, with isolated metastasis in the sternum (85\%), isolated metastasis to bone, or single lung nodular (82\%). Some were even following curative intent after multiple metastases had responded well to primary systemic therapy (29\%). The panel was split on the question whether tumour subtype would influence these decisions ( $60 \%$ yes, $40 \%$ no).

\section{COVID-19 and Breast Cancer}

The panel clearly endorsed COVID-19 vaccination for all patients before receiving chemotherapy $(87 \%)$ and for all caregivers (93\%). Asked for a prioritisation, COVID-19 vaccination should be offered primarily to all women with newly diagnosed breast cancer $(42 \%)$ and those with ongoing chemotherapy/radiotherapy (38\%). However, vaccination for patients with recently completed chemotherapy/radiotherapy was considered with less priority (21\%).

\section{Summary}

Despite having to be held fully virtual this year, the 17th St. Gallen International Breast Cancer conference was a huge success. Almost 3,500 participants from more than 100 countries worldwide participated, and lively dis- cussions as well as excellent audience interaction took place.

The 2021 Panel has found consensus on several important issues, some of them indicating the progress in the respective fields of research and clinical standards: for ipsilateral recurrence, (another) breast-conserving surgery was clearly endorsed for the first time. Also, the idea of omitting surgery after good response to neoadjuvant therapy was clearly declined. Revolutionarily, radiotherapy hypofractionation was endorsed for virtually all radiotherapy indications, which offers great potential to saving respective resources worldwide. For systemic treatment, the importance of OFS for premenopausal women with luminal breast cancer was underlined and the need for chemotherapy further reduced. The importance of pCR for approving new treatments was denied, as well as checkpoint inhibitors for triple-negative disease or pertuzumab for node-negative HER2-positive breast cancer.

While some areas of controversy persist for which no consensus could be found (platinum salts for TNBC, adjuvant CDK4/6 inhibitors, anthracyclines for HER2-positive disease, addition of adjuvant chemotherapy to OFS in premenopausal patients at moderate risk, anthracyclines for node-negative luminal breast cancer), the Consensus Panel once more re-defined the standard of care for early breast cancer. We are looking forward to both the full Consensus manuscript as well as to the 18th SGBCC to be held in Vienna in March 2023.

\section{Conflict of Interest Statement}

C.T. received compensation for advisory boards and lectures (H, SAB) from Amgen, Astra-Zeneca, Celgene, Daiichi Sankyo, Eisai, Lilly, MSD, Mylan, Nanostring, Novartis, Pfizer, Pierre Fabre, Puma, Roche, and Vifor, as well as research support (RF, by discount prizes) from American Diagnostica, Affymetrix, and Nanostring. M.B. has received personal fees and consulting fees from Amgen, AstraZeneca, Eli Lilly, MSD, Novartis, Pfizer, Roche, Samsung and Seagen. N.H. reports honoraria for lectures and/or consulting from Astra Zeneca, Daiichi-Sankyo, Lilly, MSD, Novartis, Pierre Fabre, Pfizer, Roche, and Seattle Genetics. M.G. reports personal fees/travel support from Amgen, AstraZeneca, EliLilly, Novartis; consultancy fees from DaiichiSankyo, EliLilly, Veracyte, Tolmar, LifeBrain; an immediate family member is employed by Sandoz.

\section{Funding Sources}

There was no funding for this article.

\section{Author Contributions}

All authors were involved in the writing and correcting of this article. 


\section{References}

1 Pilewskie M, Morrow M. Margins in breast cancer: how much is enough? Cancer. 2018 Apr:124(7):1335-41.

2 Curigliano G, Burstein HJ, Winer EP, Gnant M, Dubsky P, Loibl S, et al.; St. Gallen International Expert Consensus on the Primary Therapy of Early Breast Cancer 2017. De-escalating and escalating treatments for earlystage breast cancer: the St. Gallen International Expert Consensus Conference on the Primary Therapy of Early Breast Cancer 2017. Ann Oncol. 2017 Aug;28(8):1700-12.
3 Burstein HJ, Curigliano G, Loibl S, Dubsky P, Gnant M, Poortmans P, et al.; Members of the St. Gallen International Consensus Panel on the Primary Therapy of Early Breast Cancer 2019. Estimating the benefits of therapy for early-stage breast cancer: the St. Gallen International Consensus Guidelines for the primary therapy of early breast cancer 2019 . Ann Oncol. 2019 Oct;30(10):1541-57.
4 Balic M, Thomssen C, Würstlein R, Gnant M, Harbeck N. St. Gallen/Vienna 2019: A Brief Summary of the Consensus Discussion on the Optimal Primary Breast Cancer Treatment. Breast Care (Basel). 2019 Apr;14(2):103-10.

5 Harbeck N, Gnant M. Breast cancer. Lancet. 2017 Mar;389(10074):1134-50.

6 Harbeck N, Penault-Llorca F, Cortes J, Gnant M, Houssami N, Poortmans P, et al. Breast cancer. Nat Rev Dis Primers. 2019 Sep;5(1): 66. 